\title{
Effect of Pre-Harvest Foliar Spray of Micronutrients on Chemical Properties of Mango Cv. Langra at Ambient Storage Conditions
}

\author{
Rashmi Pawar ${ }^{1}$, C.P. Singh ${ }^{1}$, P.P. Singh ${ }^{2}$ and R. Meena ${ }^{3} *$ \\ ${ }^{1}$ Department of Horticulture, G.B.P.U.A. \& T., Pantnagar-263145, U.S. Nagar (U.K.), India \\ ${ }^{2}$ Deptartment of Agromateorology, JNKVV, Jabalpur (M.P.), India \\ ${ }^{3}$ Department of Soil Science and Agricultural Chemistry, Institute of Agricultural Sciences, \\ Banaras Hindu University, Varanasi- 221005 (U.P.), India \\ *Corresponding author
}

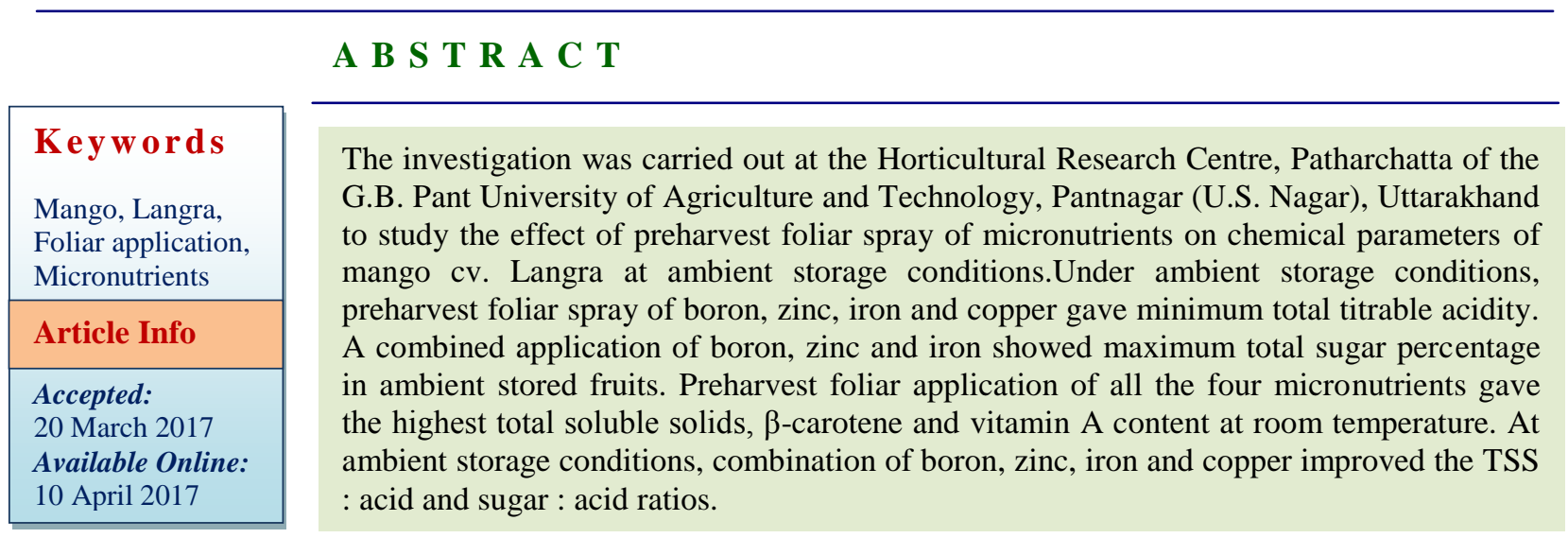

\section{Introduction}

Mango (Mangifera indica L.) the king of fruits" the main fruit of Asia and possessing own importance all over the world has been in cultivation in the Indian sub-continent for well over 4000 years (De Candolle, 1904). In India nearly 16.2 million tonnes of mangoes were produced from 2.4 million ha area. Out of the total 6.7 million hectare area, the fruit production in India is 76.4 million tonnes in 2011-12). The productivity of mango in India is low i.e. only 11.4 tonnes per hectare (Indian Horticulture Data Base, 2011-12). Mango shares 20.3 percent to total production (Indian Horticulture Data Base, 2010-11). Langra is one of the main commercial cultivars of North India. Foliar feeding is one way to achieve the goal because nutrients can be applied directly to the site of their metabolism and are, therefore, less subjected to various losses as in the case of soil application. Moreover, for rapid response and correction of deficiencies of micronutrients, foliar sprays of nutrients especially $\mathrm{Zn}, \mathrm{B}, \mathrm{Cu}$ and $\mathrm{Fe}$ have been used singly and in combination. Foliar feeding is beneficial for accelerating improvement in physico-chemical characters of fruits (Srivastava, 1993).

\section{Materials and Methods}

The investigation was carried out in the two years at the Horticulture Research Centre, of 
the University. The experiment was conducted on 27 year old trees of mango cv. Langra planted at $12 \mathrm{~m}$ distance in square system and maintained under uniform cultural practices. The trees were almost uniform in growth and vigour. The soil was well drained, sandy loam having $\mathrm{pH}$ (6.9), Zn (0.330 ppm), $\mathrm{Fe}$ (23.994 ppm), Cu (2.934 ppm) and B (1.27 $\mathrm{ppm})$. The treatment comprises of two levels of boron ( 0 and $0.4 \%)$, zinc ( 0 and $0.1 \%$ ), iron (0 and $0.02 \%)$ and copper (0 and $0.05 \%$ ). These micronutrients were sprayed through foliar application. During the month of April and May, sixteen treatment combinations were replicated thrice and laid out as per complete randomized design.

\section{Results and Discussion}

Data recorded on percent of total sugar of mango fruits kept at ambient conditions from the day of harvest to $8^{\text {th }}$ day after harvest showed that minimum content of total sugar was in control in both the years while maximum sugar content was recorded under the treatment combination having boron, zinc and iron followed by all micronutrients in the first year and only with application of all four micronutrients in the second year (Table 1). Data also revealed that there was increase in sugar content from the day of harvest to $6^{\text {th }}$ day of storage than it declined under all the treatments in both the years. It may be because of initially after harvest starch convert into sugar and after some days of storage when acidity increases it declines amount of total sugar of fruits. At over ripening stage acidity increases because sugar converts into acids (Table 1). Plants sprayed with $\mathrm{Ca}+\mathrm{B}$, were firmer; had lower TSS, higher acidity and ascorbic acid content at harvest than those in control. Similarly, such fruit after 5 days storage were firmer and brighter, and have significantly lower TSS (7.9 and 7.8\%); higher ascorbic acid content (43.7 and $45.0 \mathrm{mg} / 100 \mathrm{~g}$ pulp) and acidity
(1.08 and 1.07\%) than those in control or those receiving B alone (Singh et al., 2007).

Maximum acidity was noted under control while it was minimum in the treatment having application of all the four micronutrients (Table 2) from day of harvest to $8^{\text {th }}$ day of storage in the first and second year. We know that sugar and acidity are having reverse relationship with each other and it is clear that from the above findings in the case of total sugar (Table 1) that combination of all the four micronutrients increased the amount of sugar while in the case of total titrable acidity all the four micronutrients reduced the amount of total titrable acidity. Hence, acidity percent was declined up to $6^{\text {th }}$ day of storage under all the treatments than it showed the increased trend in all the treatments in both the years (Table 2). Similarly Sanches et al., (2005) reported that acidity of fruits decreased continuously throughout the storage period in the case of Gola variety of ber.

Data recorded in table 3 on total soluble solids of the mango fruit stored at ambient conditions showed minimum T.S.S. from day of harvest to $8^{\text {th }}$ day of storage, found in control and maximum in the treatment combination having all micronutrients in both the years respectively. Observations also revealed that the minimum T.S.S. was recorded under all the treatment on the day of harvest and it increased up to $4^{\text {th }}$ day of storage and after that it declined. Trees were sprayed with 1 per cent aqueous solution of zinc sulphate ( $\mathrm{Zn} 0.23$ per cent) once or twice (at 20 days interval) starting on 11 May and fruits were stored in a thoroughly cleaned, predisinfected and properly ventilated room for 10 days at $29-34^{\circ} \mathrm{C}$ and $85-90$ per cent RH. Two sprays of zinc sulphate showed a positive effect on the total sugar and TSS contents of fruits which continued to increase during storage. 
Table.1 Effect of preharvest foliar spray of micronutrients on total sugar of fruit at ambient storage conditions in mango cv. Langra

\begin{tabular}{|c|c|c|c|c|c|c|c|c|c|c|}
\hline \multirow{3}{*}{ Treatment } & \multicolumn{10}{|c|}{ Total sugar (\%) } \\
\hline & \multicolumn{5}{|c|}{ 2004-05 } & \multicolumn{5}{|c|}{ 2005-06 } \\
\hline & 0 day & 2 days & 4 days & 6 days & 8 days & 0 day & 2 days & 4 days & 6 days & 8 days \\
\hline $\mathrm{B}_{0} \mathrm{Zn}_{0} \mathrm{Fe}_{0} \mathrm{Cu}_{0}$ & $\begin{array}{c}9.19 \\
(17.64)\end{array}$ & $\begin{array}{c}9.29 \\
(17.74)\end{array}$ & $\begin{array}{c}9.49 \\
(17.94)\end{array}$ & $\begin{array}{c}9.52 \\
(17.97)\end{array}$ & $\begin{array}{c}9.02 \\
(17.48)\end{array}$ & $\begin{array}{c}9.57 \\
(18.02)\end{array}$ & $\begin{array}{c}9.67 \\
(18.11)\end{array}$ & $\begin{array}{c}9.87 \\
(18.31)\end{array}$ & $\begin{array}{c}9.90 \\
(18.34)\end{array}$ & $\begin{array}{c}9.40 \\
(17.85)\end{array}$ \\
\hline $\mathrm{B}_{0} \mathrm{Zn}_{0} \mathrm{Fe}_{0} \mathrm{Cu}_{1}$ & $\begin{array}{c}9.42 \\
(17.87)\end{array}$ & $\begin{array}{c}9.52 \\
(17.97)\end{array}$ & $\begin{array}{c}9.72 \\
(18.17)\end{array}$ & $\begin{array}{c}9.75 \\
(18.20)\end{array}$ & $\begin{array}{c}9.25 \\
(17.71)\end{array}$ & $\begin{array}{c}9.72 \\
(18.17)\end{array}$ & $\begin{array}{c}9.82 \\
(18.26)\end{array}$ & $\begin{array}{c}10.02 \\
(18.45)\end{array}$ & $\begin{array}{c}10.05 \\
(18.49)\end{array}$ & $\begin{array}{c}9.55 \\
(18.00)\end{array}$ \\
\hline $\mathrm{B}_{0} \mathrm{Zn}_{0} \mathrm{Fe}_{1} \mathrm{Cu}_{0}$ & $\begin{array}{c}9.56 \\
18.01)\end{array}$ & $\begin{array}{c}9.66 \\
(18.11)\end{array}$ & $\begin{array}{c}9.86 \\
(18.30)\end{array}$ & $\begin{array}{c}9.89 \\
(18.33)\end{array}$ & $\begin{array}{c}9.39 \\
(17.85)\end{array}$ & $\begin{array}{c}9.86 \\
(18.30)\end{array}$ & $\begin{array}{c}9.96 \\
(18.39)\end{array}$ & $\begin{array}{c}10.16 \\
(18.58)\end{array}$ & $\begin{array}{c}10.19 \\
(18.62)\end{array}$ & $\begin{array}{c}9.69 \\
(18.14)\end{array}$ \\
\hline $\mathrm{B}_{0} \mathrm{Zn}_{0} \mathrm{Fe}_{1} \mathrm{Cu}_{1}$ & $\begin{array}{c}9.72 \\
(18.17)\end{array}$ & $\begin{array}{c}9.82 \\
(18.27)\end{array}$ & $\begin{array}{c}10.02 \\
(18.46)\end{array}$ & $\begin{array}{c}10.02 \\
(18.46)\end{array}$ & $\begin{array}{c}9.52 \\
(17.97)\end{array}$ & $\begin{array}{c}9.94 \\
(18.37)\end{array}$ & $\begin{array}{c}10.04 \\
(18.47)\end{array}$ & $\begin{array}{c}10.24 \\
(18.66)\end{array}$ & $\begin{array}{c}10.24 \\
(18.66)\end{array}$ & $\begin{array}{c}9.74 \\
(18.18)\end{array}$ \\
\hline $\mathrm{B}_{0} \mathrm{Zn}_{1} \mathrm{Fe}_{0} \mathrm{Cu}_{0}$ & $\begin{array}{c}9.84 \\
(18.28)\end{array}$ & $\begin{array}{c}9.94 \\
(18.38)\end{array}$ & $\begin{array}{c}10.14 \\
(18.57)\end{array}$ & $\begin{array}{c}10.18 \\
(18.60)\end{array}$ & $\begin{array}{c}9.68 \\
(18.12)\end{array}$ & $\begin{array}{c}10.24 \\
(18.67)\end{array}$ & $\begin{array}{c}10.31 \\
(18.79)\end{array}$ & $\begin{array}{c}10.54 \\
(18.95)\end{array}$ & $\begin{array}{c}10.54 \\
(18.95)\end{array}$ & $\begin{array}{c}10.04 \\
(18.48)\end{array}$ \\
\hline $\mathrm{B}_{0} \mathrm{Zn}_{1} \mathrm{Fe}_{0} \mathrm{Cu}_{1}$ & $\begin{array}{c}9.93 \\
(18.37)\end{array}$ & $\begin{array}{c}10.03 \\
(18.46)\end{array}$ & $\begin{array}{c}10.23 \\
(18.65)\end{array}$ & $\begin{array}{c}10.23 \\
(18.65)\end{array}$ & $\begin{array}{c}9.73 \\
(18.18)\end{array}$ & $\begin{array}{c}10.28 \\
(18.70)\end{array}$ & $\begin{array}{c}10.38 \\
(18.79)\end{array}$ & $\begin{array}{c}10.58 \\
(18.98)\end{array}$ & $\begin{array}{c}10.61 \\
(19.01)\end{array}$ & $\begin{array}{c}10.11 \\
(18.54)\end{array}$ \\
\hline $\mathrm{B}_{0} \mathrm{Zn}_{1} \mathrm{Fe}_{1} \mathrm{Cu}_{0}$ & $\begin{array}{c}10.09 \\
(18.52)\end{array}$ & $\begin{array}{c}10.19 \\
(18.61)\end{array}$ & $\begin{array}{c}10.39 \\
(18.80)\end{array}$ & $\begin{array}{c}10.42 \\
(18.83)\end{array}$ & $\begin{array}{c}9.92 \\
(18.36)\end{array}$ & $\begin{array}{c}10.44 \\
(18.85)\end{array}$ & $\begin{array}{c}10.54 \\
(18.95)\end{array}$ & $\begin{array}{c}10.74 \\
(19.13)\end{array}$ & $\begin{array}{c}10.81 \\
(19.19)\end{array}$ & $\begin{array}{c}10.31 \\
(18.73)\end{array}$ \\
\hline $\mathrm{B}_{0} \mathrm{Zn}_{1} \mathrm{Fe}_{1} \mathrm{Cu}_{1}$ & $\begin{array}{c}10.17 \\
(18.59)\end{array}$ & $\begin{array}{c}10.27 \\
(18.69)\end{array}$ & $\begin{array}{c}10.47 \\
(18.88)\end{array}$ & $\begin{array}{c}10.47 \\
(18.88)\end{array}$ & $\begin{array}{c}9.97 \\
(18.40)\end{array}$ & $\begin{array}{c}10.51 \\
(18.91)\end{array}$ & $\begin{array}{c}10.61 \\
(19.01)\end{array}$ & $\begin{array}{c}10.81 \\
(19.19)\end{array}$ & $\begin{array}{c}10.84 \\
(19.22)\end{array}$ & $\begin{array}{c}10.34 \\
(18.76)\end{array}$ \\
\hline $\mathrm{B}_{1} \mathrm{Zn}_{0} \mathrm{Fe}_{0} \mathrm{Cu}_{0}$ & $\begin{array}{c}10.19 \\
(18.61)\end{array}$ & $\begin{array}{c}10.29 \\
(18.71)\end{array}$ & $\begin{array}{c}10.49 \\
(18.90)\end{array}$ & $\begin{array}{c}10.55 \\
(18.96)\end{array}$ & $\begin{array}{c}10.05 \\
(18.49)\end{array}$ & $\begin{array}{c}10.53 \\
(18.94)\end{array}$ & $\begin{array}{c}10.63 \\
(19.03)\end{array}$ & $\begin{array}{c}10.83 \\
(19.22)\end{array}$ & $\begin{array}{c}10.83 \\
(19.22)\end{array}$ & $\begin{array}{c}10.33 \\
(18.75)\end{array}$ \\
\hline $\mathrm{B}_{1} \mathrm{Zn}_{0} \mathrm{Fe}_{0} \mathrm{Cu}_{1}$ & $\begin{array}{c}10.24 \\
(18.66)\end{array}$ & $\begin{array}{c}10.34 \\
(18.75)\end{array}$ & $\begin{array}{c}10.54 \\
(18.94)\end{array}$ & $\begin{array}{c}10.54 \\
(18.94)\end{array}$ & $\begin{array}{c}10.04 \\
(18.47)\end{array}$ & $\begin{array}{c}10.54 \\
(18.94)\end{array}$ & $\begin{array}{c}10.64 \\
(19.03)\end{array}$ & $\begin{array}{c}10.84 \\
(19.22)\end{array}$ & $\begin{array}{c}10.87 \\
(19.25)\end{array}$ & $\begin{array}{c}10.37 \\
(18.79)\end{array}$ \\
\hline $\mathrm{B}_{1} \mathrm{Zn}_{0} \mathrm{Fe}_{1} \mathrm{Cu}_{0}$ & $\begin{array}{c}10.27 \\
(18.69)\end{array}$ & $\begin{array}{c}10.37 \\
(18.78)\end{array}$ & $\begin{array}{c}10.57 \\
(18.97)\end{array}$ & $\begin{array}{c}10.60 \\
(19.00)\end{array}$ & $\begin{array}{c}10.10 \\
(18.53)\end{array}$ & $\begin{array}{c}11.17 \\
(19.53)\end{array}$ & $\begin{array}{c}11.27 \\
(19.62)\end{array}$ & $\begin{array}{c}11.47 \\
(19.80)\end{array}$ & $\begin{array}{c}11.57 \\
(19.89)\end{array}$ & $\begin{array}{c}11.07 \\
(19.44)\end{array}$ \\
\hline $\mathrm{B}_{1} \mathrm{Zn}_{0} \mathrm{Fe}_{1} \mathrm{Cu}_{1}$ & $\begin{array}{c}10.37 \\
(18.78)\end{array}$ & $\begin{array}{c}10.47 \\
(18.88)\end{array}$ & $\begin{array}{c}10.67 \\
(19.06)\end{array}$ & $\begin{array}{c}10.73 \\
(19.12)\end{array}$ & $\begin{array}{c}10.23 \\
(18.66)\end{array}$ & $\begin{array}{c}11.21 \\
(19.56)\end{array}$ & $\begin{array}{c}11.31 \\
(19.66)\end{array}$ & $\begin{array}{c}11.51 \\
(19.84)\end{array}$ & $\begin{array}{c}11.51 \\
(19.84)\end{array}$ & $\begin{array}{c}11.01 \\
(19.38)\end{array}$ \\
\hline $\mathrm{B}_{1} \mathrm{Zn}_{1} \mathrm{Fe}_{0} \mathrm{Cu}_{0}$ & $\begin{array}{c}10.44 \\
(18.85)\end{array}$ & $\begin{array}{c}10.54 \\
(18.94)\end{array}$ & $\begin{array}{c}10.74 \\
(19.13)\end{array}$ & $\begin{array}{c}10.80 \\
(19.19)\end{array}$ & $\begin{array}{c}10.30 \\
(18.72)\end{array}$ & $\begin{array}{c}11.29 \\
(19.64)\end{array}$ & $\begin{array}{c}11.39 \\
(19.73)\end{array}$ & $\begin{array}{c}11.59 \\
(19.91)\end{array}$ & $\begin{array}{c}11.63 \\
(19.94)\end{array}$ & $\begin{array}{c}11.13 \\
(19.49)\end{array}$ \\
\hline $\mathrm{B}_{1} \mathrm{Zn}_{1} \mathrm{Fe}_{0} \mathrm{Cu}_{1}$ & $\begin{array}{c}10.53 \\
(18.93)\end{array}$ & $\begin{array}{c}10.63 \\
(19.03)\end{array}$ & $\begin{array}{c}10.83 \\
(19.21)\end{array}$ & $\begin{array}{c}10.86 \\
(19.24)\end{array}$ & $\begin{array}{c}10.36 \\
(18.78)\end{array}$ & $\begin{array}{c}11.63 \\
(19.94)\end{array}$ & $\begin{array}{c}11.73 \\
(20.03)\end{array}$ & $\begin{array}{c}11.93 \\
(20.21)\end{array}$ & $\begin{array}{c}12.00 \\
(20.26)\end{array}$ & $\begin{array}{c}11.50 \\
(19.82)\end{array}$ \\
\hline $\mathrm{B}_{1} \mathrm{Zn}_{1} \mathrm{Fe}_{1} \mathrm{Cu}_{0}$ & $\begin{array}{c}10.81 \\
(19.20)\end{array}$ & $\begin{array}{c}10.91 \\
(19.29)\end{array}$ & $\begin{array}{c}11.11 \\
(19.47)\end{array}$ & $\begin{array}{c}11.15 \\
(19.50)\end{array}$ & $\begin{array}{c}10.65 \\
(19.04)\end{array}$ & $\begin{array}{c}12.13 \\
(20.38)\end{array}$ & $\begin{array}{c}12.23 \\
(20.47)\end{array}$ & $\begin{array}{c}12.43 \\
(20.64)\end{array}$ & $\begin{array}{c}12.46 \\
(20.67)\end{array}$ & $\begin{array}{c}11.96 \\
(20.24)\end{array}$ \\
\hline $\mathrm{B}_{1} \mathrm{Zn}_{1} \mathrm{Fe}_{1} \mathrm{Cu}_{1}$ & $\begin{array}{c}11.16 \\
(19.52)\end{array}$ & $\begin{array}{c}11.26 \\
(19.61)\end{array}$ & $\begin{array}{c}11.46 \\
(19.79)\end{array}$ & $\begin{array}{c}11.56 \\
(19.88)\end{array}$ & $\begin{array}{c}11.06 \\
(19.43)\end{array}$ & $\begin{array}{l}12.20 \\
(20.45)\end{array}$ & $\begin{array}{c}12.30 \\
(20.53)\end{array}$ & $\begin{array}{c}12.50 \\
(20.71)\end{array}$ & $\begin{array}{c}12.54 \\
(20.74)\end{array}$ & $\begin{array}{c}12.04 \\
(20.30)\end{array}$ \\
\hline S.Em. \pm & $\begin{array}{c}0.69 \\
(0.35)\end{array}$ & $\begin{array}{c}0.68 \\
(0.35)\end{array}$ & $\begin{array}{c}0.67 \\
(0.34)\end{array}$ & $\begin{array}{c}0.86 \\
(0.45)\end{array}$ & $\begin{array}{c}0.90 \\
(0.47)\end{array}$ & $\begin{array}{c}0.74 \\
(0.38)\end{array}$ & $\begin{array}{c}1.20 \\
(0.63)\end{array}$ & $\begin{array}{c}0.72 \\
(0.37)\end{array}$ & $\begin{array}{c}0.89 \\
(0.46)\end{array}$ & $\begin{array}{c}0.93 \\
(0.48)\end{array}$ \\
\hline C.D. at $5 \%$ & $\begin{array}{c}0.45 \\
(0.44)\end{array}$ & $\begin{array}{c}0.45 \\
(0.44)\end{array}$ & $\begin{array}{c}0.45 \\
(0.44)\end{array}$ & $\begin{array}{c}0.51 \\
(0.51)\end{array}$ & $\begin{array}{c}0.52 \\
(0.51)\end{array}$ & $\begin{array}{c}0.48 \\
(0.46)\end{array}$ & $\begin{array}{c}0.61 \\
(0.60)\end{array}$ & $\begin{array}{c}0.48 \\
(0.46)\end{array}$ & $\begin{array}{c}0.54 \\
(0.51)\end{array}$ & $\begin{array}{c}0.54 \\
(0.52)\end{array}$ \\
\hline
\end{tabular}

Data in parentheses indicate transformed value 
Table.2 Effect of pre-harvest foliar spray of micronutrients on total titrable acidity at ambient storage conditions in mango fruit $\mathrm{cv}$. Langra

\begin{tabular}{|c|c|c|c|c|c|c|c|c|c|c|}
\hline \multirow{3}{*}{ Treatment } & \multicolumn{10}{|c|}{ Total titrable acidity $(\%)$} \\
\hline & \multicolumn{5}{|c|}{ 2004-05 } & \multicolumn{5}{|c|}{ 2005-06 } \\
\hline & 0 day & 2 days & 4 days & 6 days & 8 days & 0 day & 2 days & 4 days & 6 days & 8 days \\
\hline $\mathrm{B}_{0} \mathrm{Zn}_{0} \mathrm{Fe}_{0} \mathrm{Cu}_{0}$ & $\begin{array}{c}0.226 \\
(2.727)\end{array}$ & $\begin{array}{c}0.205 \\
(2.603)\end{array}$ & $\begin{array}{c}0.186 \\
(2.473)\end{array}$ & $\begin{array}{c}0.185 \\
(2.473)\end{array}$ & $\begin{array}{c}0.386 \\
(3.563)\end{array}$ & $\begin{array}{c}0.222 \\
(2.702)\end{array}$ & $\begin{array}{c}0.203 \\
(2.578)\end{array}$ & $\begin{array}{c}0.183 \\
(2.447)\end{array}$ & $\begin{array}{c}0.182 \\
(2.447)\end{array}$ & $\begin{array}{c}0.380 \\
(3.545)\end{array}$ \\
\hline $\mathrm{B}_{0} \mathrm{Zn}_{0} \mathrm{Fe}_{0} \mathrm{Cu}_{1}$ & $\begin{array}{c}0.222 \\
(2.700)\end{array}$ & $\begin{array}{c}0.203 \\
(2.574)\end{array}$ & $\begin{array}{c}0.182 \\
(2.442)\end{array}$ & $\begin{array}{c}0.178 \\
(2.420)\end{array}$ & $\begin{array}{c}0.378 \\
(3.526)\end{array}$ & $\begin{array}{c}0.222 \\
(2.702)\end{array}$ & $\begin{array}{c}0.202 \\
(2.578)\end{array}$ & $\begin{array}{c}0.183 \\
(2.447)\end{array}$ & $\begin{array}{c}0.179 \\
(2.424)\end{array}$ & $\begin{array}{c}0.379 \\
(3.529)\end{array}$ \\
\hline $\mathrm{B}_{0} \mathrm{Zn}_{0} \mathrm{Fe}_{1} \mathrm{Cu}_{0}$ & $\begin{array}{c}0.213 \\
(2.647)\end{array}$ & $\begin{array}{c}0.193 \\
(2.520)\end{array}$ & $\begin{array}{c}0.173 \\
(2.386)\end{array}$ & $\begin{array}{c}0.171 \\
(2.362)\end{array}$ & $\begin{array}{c}0.370 \\
(3.487)\end{array}$ & $\begin{array}{c}0.215 \\
(2.654)\end{array}$ & $\begin{array}{c}0.195 \\
(2.526)\end{array}$ & $\begin{array}{c}0.174 \\
(2.393)\end{array}$ & $\begin{array}{c}0.171 \\
(2.369)\end{array}$ & $\begin{array}{c}0.371 \\
(3.492)\end{array}$ \\
\hline $\mathrm{B}_{0} \mathrm{Zn}_{0} \mathrm{Fe}_{1} \mathrm{Cu}_{1}$ & $\begin{array}{c}0.212 \\
(2.641)\end{array}$ & $\begin{array}{c}0.191 \\
(2.513)\end{array}$ & $\begin{array}{c}0.172 \\
(2.379)\end{array}$ & $\begin{array}{c}0.169 \\
(2.355)\end{array}$ & $\begin{array}{c}0.369 \\
(3.482)\end{array}$ & $\begin{array}{c}0.213 \\
(2.647)\end{array}$ & $\begin{array}{c}0.194 \\
(2.520)\end{array}$ & $\begin{array}{c}0.173 \\
(2.386)\end{array}$ & $\begin{array}{c}0.170 \\
(2.362)\end{array}$ & $\begin{array}{c}0.370 \\
(3.487)\end{array}$ \\
\hline $\mathrm{B}_{0} \mathrm{Zn}_{1} \mathrm{Fe}_{0} \mathrm{Cu}_{0}$ & $\begin{array}{c}0.209 \\
(2.620)\end{array}$ & $\begin{array}{c}0.188 \\
(2.491)\end{array}$ & $\begin{array}{c}0.170 \\
(2.355)\end{array}$ & $\begin{array}{c}0.169 \\
(2.355)\end{array}$ & $\begin{array}{c}0.369 \\
(3.482)\end{array}$ & $\begin{array}{c}0.208 \\
(2.614)\end{array}$ & $\begin{array}{c}0.188 \\
(2.484)\end{array}$ & $\begin{array}{c}0.168 \\
(2.348)\end{array}$ & $\begin{array}{c}0.168 \\
(2.348)\end{array}$ & $\begin{array}{c}0.368 \\
(3.478)\end{array}$ \\
\hline $\mathrm{B}_{0} \mathrm{Zn}_{1} \mathrm{Fe}_{0} \mathrm{Cu}_{1}$ & $\begin{array}{c}0.207 \\
(2.609)\end{array}$ & $\begin{array}{c}0.187 \\
(2.480)\end{array}$ & $\begin{array}{c}0.168 \\
(2.344)\end{array}$ & $\begin{array}{c}0.167 \\
(2.344)\end{array}$ & $\begin{array}{c}0.366 \\
(3.474)\end{array}$ & $\begin{array}{c}0.207 \\
(2.607)\end{array}$ & $\begin{array}{c}0.186 \\
(2.478)\end{array}$ & $\begin{array}{c}0.166 \\
(2.341)\end{array}$ & $\begin{array}{c}0.167 \\
(2.341)\end{array}$ & $\begin{array}{c}0.367 \\
(3.473)\end{array}$ \\
\hline $\mathrm{B}_{0} \mathrm{Zn}_{1} \mathrm{Fe}_{1} \mathrm{Cu}_{0}$ & $\begin{array}{c}0.205 \\
(2.600)\end{array}$ & $\begin{array}{c}0.186 \\
(2.467)\end{array}$ & $\begin{array}{c}0.164 \\
(2.329)\end{array}$ & $\begin{array}{c}0.165 \\
(2.329)\end{array}$ & $\begin{array}{c}0.364 \\
(3.465)\end{array}$ & $\begin{array}{c}0.201 \\
(2.571)\end{array}$ & $\begin{array}{c}0.181 \\
(2.440)\end{array}$ & $\begin{array}{c}0.163 \\
(2.301)\end{array}$ & $\begin{array}{c}0.161 \\
(2.301)\end{array}$ & $\begin{array}{c}0.360 \\
(3.446)\end{array}$ \\
\hline $\mathrm{B}_{0} \mathrm{Zn}_{1} \mathrm{Fe}_{1} \mathrm{Cu}_{1}$ & $\begin{array}{c}0.200 \\
(2.560)\end{array}$ & $\begin{array}{c}0.182 \\
(2.428)\end{array}$ & $\begin{array}{c}0.160 \\
(2.289)\end{array}$ & $\begin{array}{c}0.156 \\
(2.264)\end{array}$ & $\begin{array}{c}0.356 \\
(3.422)\end{array}$ & $\begin{array}{c}0.200 \\
(2.562)\end{array}$ & $\begin{array}{c}0.179 \\
(2.430)\end{array}$ & $\begin{array}{c}0.160 \\
(2.291)\end{array}$ & $\begin{array}{c}0.157 \\
(2.266)\end{array}$ & $\begin{array}{c}0.359 \\
(3.423)\end{array}$ \\
\hline $\mathrm{B}_{1} \mathrm{Zn}_{0} \mathrm{Fe}_{0} \mathrm{Cu}_{0}$ & $\begin{array}{c}0.196 \\
(2.544)\end{array}$ & $\begin{array}{c}0.178 \\
(2.411)\end{array}$ & $\begin{array}{c}0.160 \\
(2.295)\end{array}$ & $\begin{array}{c}0.157 \\
(2.270)\end{array}$ & $\begin{array}{c}0.357 \\
(3.425)\end{array}$ & $\begin{array}{c}0.198 \\
(2.548)\end{array}$ & $\begin{array}{c}0.178 \\
(2.415)\end{array}$ & $\begin{array}{c}0.158 \\
(2.275)\end{array}$ & $\begin{array}{c}0.158 \\
(2.275)\end{array}$ & $\begin{array}{c}0.357 \\
(3.429)\end{array}$ \\
\hline $\mathrm{B}_{1} \mathrm{Zn}_{0} \mathrm{Fe}_{0} \mathrm{Cu}_{1}$ & $\begin{array}{c}0.192 \\
(2.508)\end{array}$ & $\begin{array}{c}0.173 \\
(2.374)\end{array}$ & $\begin{array}{c}0.152 \\
(2.231)\end{array}$ & $\begin{array}{c}0.148 \\
(2.205)\end{array}$ & $\begin{array}{c}0.349 \\
(3.383)\end{array}$ & $\begin{array}{c}0.192 \\
(2.513)\end{array}$ & $\begin{array}{c}0.166 \\
(2.331)\end{array}$ & $\begin{array}{c}0.146 \\
(2.185)\end{array}$ & $\begin{array}{c}0.143 \\
(2.159)\end{array}$ & $\begin{array}{c}0.342 \\
(3.353)\end{array}$ \\
\hline $\mathrm{B}_{1} \mathrm{Zn}_{0} \mathrm{Fe}_{1} \mathrm{Cu}_{0}$ & $\begin{array}{c}0.191 \\
(2.502)\end{array}$ & $\begin{array}{c}0.172 \\
(2.366)\end{array}$ & $\begin{array}{c}0.151 \\
(2.223)\end{array}$ & $\begin{array}{c}0.147 \\
(2.197)\end{array}$ & $\begin{array}{c}0.348 \\
(3.378)\end{array}$ & $\begin{array}{c}0.191 \\
(2.506)\end{array}$ & $\begin{array}{c}0.165 \\
(2.323)\end{array}$ & $\begin{array}{c}0.145 \\
(2.177)\end{array}$ & $\begin{array}{c}0.141 \\
(2.151)\end{array}$ & $\begin{array}{c}0.341 \\
(3.348)\end{array}$ \\
\hline $\mathrm{B}_{1} \mathrm{Zn}_{0} \mathrm{Fe}_{1} \mathrm{Cu}_{1}$ & $\begin{array}{c}0.190 \\
(2.500)\end{array}$ & $\begin{array}{c}0.171 \\
(2.365)\end{array}$ & $\begin{array}{c}0.150 \\
(2.222)\end{array}$ & $\begin{array}{c}0.147 \\
(2.197)\end{array}$ & $\begin{array}{c}0.346 \\
(3.377)\end{array}$ & $\begin{array}{c}0.189 \\
(2.500)\end{array}$ & $\begin{array}{c}0.170 \\
(2.365)\end{array}$ & $\begin{array}{c}0.152 \\
(2.222)\end{array}$ & $\begin{array}{c}0.145 \\
(2.197)\end{array}$ & $\begin{array}{c}0.347 \\
(3.377)\end{array}$ \\
\hline $\mathrm{B}_{1} \mathrm{Zn}_{1} \mathrm{Fe}_{0} \mathrm{Cu}_{0}$ & $\begin{array}{c}0.188 \\
(2.476)\end{array}$ & $\begin{array}{c}0.166 \\
(2.339)\end{array}$ & $\begin{array}{c}0.145 \\
(2.194)\end{array}$ & $\begin{array}{c}0.143 \\
(2.170)\end{array}$ & $\begin{array}{c}0.342 \\
(3.359)\end{array}$ & $\begin{array}{c}0.186 \\
(2.469)\end{array}$ & $\begin{array}{c}0.166 \\
(2.332)\end{array}$ & $\begin{array}{c}0.146 \\
(2.187)\end{array}$ & $\begin{array}{c}0.142 \\
(2.162)\end{array}$ & $\begin{array}{c}0.343 \\
(3.354)\end{array}$ \\
\hline $\mathrm{B}_{1} \mathrm{Zn}_{1} \mathrm{Fe}_{0} \mathrm{Cu}_{1}$ & $\begin{array}{c}0.184 \\
(2.456)\end{array}$ & $\begin{array}{c}0.164 \\
(2.318)\end{array}$ & $\begin{array}{c}0.144 \\
(2.172)\end{array}$ & $\begin{array}{c}0.134 \\
(2.095)\end{array}$ & $\begin{array}{c}0.335 \\
(3.311)\end{array}$ & $\begin{array}{c}0.183 \\
(2.450)\end{array}$ & $\begin{array}{c}0.162 \\
(2.312)\end{array}$ & $\begin{array}{c}0.141 \\
(2.160)\end{array}$ & $\begin{array}{c}0.140 \\
(2.147)\end{array}$ & $\begin{array}{c}0.340 \\
(3.344)\end{array}$ \\
\hline $\mathrm{B}_{1} \mathrm{Zn}_{1} \mathrm{Fe}_{1} \mathrm{Cu}_{0}$ & $\begin{array}{c}0.183 \\
(2.452)\end{array}$ & $\begin{array}{c}0.163 \\
(2.314)\end{array}$ & $\begin{array}{l}0.143 \\
(2.167)\end{array}$ & $\begin{array}{c}0.133 \\
(2.090)\end{array}$ & $\begin{array}{c}0.336 \\
(3.308)\end{array}$ & $\begin{array}{c}0.182 \\
(2.443)\end{array}$ & $\begin{array}{c}0.161 \\
(2.300)\end{array}$ & $\begin{array}{c}0.140 \\
(2.150)\end{array}$ & $\begin{array}{c}0.131 \\
(2.082)\end{array}$ & $\begin{array}{c}0.333 \\
(3.306)\end{array}$ \\
\hline $\mathrm{B}_{1} \mathrm{Zn}_{1} \mathrm{Fe}_{1} \mathrm{Cu}_{1}$ & $\begin{array}{c}0.183 \\
(2.451)\end{array}$ & $\begin{array}{c}0.163 \\
(2.314)\end{array}$ & $\begin{array}{c}0.143 \\
(2.167)\end{array}$ & $\begin{array}{c}0.140 \\
(2.142)\end{array}$ & $\begin{array}{c}0.339 \\
(3.341)\end{array}$ & $\begin{array}{c}0.182 \\
(2.443)\end{array}$ & $\begin{array}{c}0.160 \\
(2.304)\end{array}$ & $\begin{array}{c}0.142 \\
(2.157)\end{array}$ & $\begin{array}{c}0.133 \\
(2.079)\end{array}$ & $\begin{array}{c}0.332 \\
(3.301)\end{array}$ \\
\hline S.Em. \pm & $\begin{array}{c}4.41 \\
(2.20)\end{array}$ & $\begin{array}{l}4.90 \\
(2.44)\end{array}$ & $\begin{array}{c}5.41 \\
(2.70)\end{array}$ & $\begin{array}{c}6.82 \\
(3.42)\end{array}$ & $\begin{array}{c}3.00 \\
(1.50)\end{array}$ & $\begin{array}{c}4.45 \\
(2.22)\end{array}$ & $\begin{array}{c}5.42 \\
(2.72)\end{array}$ & $\begin{array}{l}6.10 \\
(3.0)\end{array}$ & $\begin{array}{c}7.10 \\
(3.55)\end{array}$ & $\begin{array}{c}3.10 \\
(1.55)\end{array}$ \\
\hline C.D. at $5 \%$ & $\begin{array}{c}0.16 \\
(0.40)\end{array}$ & $\begin{array}{c}0.16 \\
(0.41)\end{array}$ & $\begin{array}{c}0.17 \\
(0.42)\end{array}$ & $\begin{array}{c}0.17 \\
(0.47)\end{array}$ & $\begin{array}{c}0.15 \\
(0.38)\end{array}$ & $\begin{array}{c}0.16 \\
(0.41)\end{array}$ & $\begin{array}{c}0.16 \\
(0.44)\end{array}$ & $\begin{array}{c}0.17 \\
(0.45)\end{array}$ & $\begin{array}{c}0.18 \\
(0.48)\end{array}$ & $\begin{array}{c}0.18 \\
(0.39)\end{array}$ \\
\hline
\end{tabular}

Data in parentheses indicate transformed value 
Table.3 Effect of pre-harvest foliar spray of micronutrients on total soluble solids at ambient storage conditions in mango fruit cv. Langra

\begin{tabular}{|c|c|c|c|c|c|c|c|c|c|c|}
\hline \multirow{3}{*}{ Treatment } & \multicolumn{10}{|c|}{ Total soluble solids $\left({ }^{0} \mathbf{B}\right)$} \\
\hline & \multicolumn{5}{|c|}{ 2004-05 } & \multicolumn{5}{|c|}{ 2005-06 } \\
\hline & 0 day & 2 days & 4 days & 6 days & 8 days & 0 day & 2 days & 4 days & 6 days & 8 days \\
\hline $\mathrm{B}_{0} \mathrm{Zn}_{0} \mathrm{Fe}_{0} \mathrm{Cu}_{0}$ & 16.39 & 17.37 & 19.37 & 18.05 & 15.39 & 16.72 & 17.72 & 19.71 & 18.70 & 15.72 \\
\hline $\mathrm{B}_{0} \mathrm{Zn}_{0} \mathrm{Fe}_{0} \mathrm{Cu}_{1}$ & 16.40 & 17.39 & 19.38 & 18.39 & 15.41 & 16.79 & 17.79 & 19.80 & 18.79 & 15.80 \\
\hline $\mathrm{B}_{0} \mathrm{Zn}_{0} \mathrm{Fe}_{1} \mathrm{Cu}_{0}$ & 16.72 & 17.72 & 19.40 & 18.39 & 15.72 & 16.92 & 17.92 & 19.92 & 18.92 & 15.91 \\
\hline $\mathrm{B}_{0} \mathrm{Zn}_{0} \mathrm{Fe}_{1} \mathrm{Cu}_{1}$ & 16.76 & 17.72 & 19.41 & 18.72 & 15.72 & 16.99 & 17.99 & 20.00 & 18.99 & 16.00 \\
\hline $\mathrm{B}_{0} \mathrm{Zn}_{1} \mathrm{Fe}_{0} \mathrm{Cu}_{0}$ & 16.78 & 18.05 & 19.72 & 18.72 & 15.74 & 17.05 & 18.05 & 20.05 & 19.05 & 16.05 \\
\hline $\mathrm{B}_{0} \mathrm{Zn}_{1} \mathrm{Fe}_{0} \mathrm{Cu}_{1}$ & 17.00 & 17.72 & 19.72 & 19.05 & 16.05 & 17.06 & 18.05 & 20.05 & 19.05 & 16.05 \\
\hline $\mathrm{B}_{0} \mathrm{Zn}_{1} \mathrm{Fe}_{1} \mathrm{Cu}_{0}$ & 17.05 & 18.10 & 20.04 & 19.08 & 16.05 & 17.05 & 18.06 & 20.06 & 19.06 & 16.07 \\
\hline $\mathrm{B}_{0} \mathrm{Zn}_{1} \mathrm{Fe}_{1} \mathrm{Cu}_{1}$ & 17.08 & 18.05 & 20.05 & 19.05 & 16.39 & 17.39 & 18.39 & 20.35 & 19.35 & 16.39 \\
\hline $\mathrm{B}_{1} \mathrm{Zn}_{0} \mathrm{Fe}_{0} \mathrm{Cu}_{0}$ & 17.39 & 18.39 & 20.05 & 19.39 & 16.05 & 17.53 & 18.50 & 20.39 & 19.39 & 16.35 \\
\hline $\mathrm{B}_{1} \mathrm{Zn}_{0} \mathrm{Fe}_{0} \mathrm{Cu}_{1}$ & 17.42 & 18.42 & 20.39 & 19.39 & 16.39 & 17.56 & 18.53 & 20.53 & 19.50 & 16.50 \\
\hline $\mathrm{B}_{1} \mathrm{Zn}_{0} \mathrm{Fe}_{1} \mathrm{Cu}_{0}$ & 17.72 & 18.72 & 20.39 & 19.72 & 16.72 & 17.50 & 18.56 & 20.82 & 19.53 & 16.53 \\
\hline $\mathrm{B}_{1} \mathrm{Zn}_{0} \mathrm{Fe}_{1} \mathrm{Cu}_{1}$ & 18.05 & 19.05 & 20.72 & 19.72 & 16.72 & 17.82 & 18.82 & 21.06 & 19.82 & 16.82 \\
\hline $\mathrm{B}_{1} \mathrm{Zn}_{1} \mathrm{Fe}_{0} \mathrm{Cu}_{0}$ & 18.39 & 19.11 & 21.05 & 20.05 & 17.05 & 18.06 & 19.06 & 21.72 & 20.06 & 17.06 \\
\hline $\mathrm{B}_{1} \mathrm{Zn}_{1} \mathrm{Fe}_{0} \mathrm{Cu}_{1}$ & 18.70 & 19.39 & 21.39 & 20.40 & 17.39 & 18.72 & 19.72 & 21.72 & 20.72 & 17.72 \\
\hline $\mathrm{B}_{1} \mathrm{Zn}_{1} \mathrm{Fe}_{1} \mathrm{Cu}_{0}$ & 18.72 & 19.72 & 21.73 & 20.72 & 17.70 & 19.39 & 20.37 & 22.39 & 21.38 & 18.38 \\
\hline $\mathrm{B}_{1} \mathrm{Zn}_{1} \mathrm{Fe}_{1} \mathrm{Cu}_{1}$ & 19.39 & 20.38 & 22.40 & 21.39 & 18.37 & 19.40 & 20.39 & 22.40 & 21.40 & 18.41 \\
\hline S.Em. \pm & 5.15 & 4.87 & 4.39 & 5.72 & 6.77 & 5.21 & 4.93 & 4.54 & 4.77 & 5.64 \\
\hline C.D. at $5 \%$ & 1.62 & 1.62 & 1.62 & 1.81 & 1.81 & 1.64 & 1.64 & 1.64 & 1.66 & 1.67 \\
\hline
\end{tabular}


Table.4 Effect of pre-harvest foliar spray of micronutrients on $\beta$-carotene at ambient storage conditions in mango fruit cv. Langra

\begin{tabular}{|c|c|c|c|c|c|c|c|c|c|c|}
\hline \multirow{3}{*}{ Treatment } & \multicolumn{10}{|c|}{$\beta$-carotene (mg/ $100 \mathrm{~g}$ of pulp) } \\
\hline & \multicolumn{5}{|c|}{ 2004-05 } & \multicolumn{5}{|c|}{ 2005-06 } \\
\hline & 0 day & 2 days & 4 days & 6 days & 8 days & 0 day & 2 days & 4 days & 6 days & 8 days \\
\hline $\mathrm{B}_{0} \mathrm{Zn}_{0} \mathrm{Fe}_{0} \mathrm{Cu}_{0}$ & 0.95 & 1.14 & 1.44 & 1.63 & 1.54 & 0.96 & 1.18 & 1.49 & 1.68 & 1.57 \\
\hline $\mathrm{B}_{0} \mathrm{Zn}_{0} \mathrm{Fe}_{0} \mathrm{Cu}_{1}$ & 0.99 & 1.19 & 1.50 & 1.69 & 1.60 & 1.02 & 1.23 & 1.52 & 1.73 & 1.63 \\
\hline $\mathrm{B}_{0} \mathrm{Zn}_{0} \mathrm{Fe}_{1} \mathrm{Cu}_{0}$ & 1.00 & 1.20 & 1.49 & 1.70 & 1.59 & 1.03 & 1.24 & 1.55 & 1.73 & 1.64 \\
\hline $\mathrm{B}_{0} \mathrm{Zn}_{0} \mathrm{Fe}_{1} \mathrm{Cu}_{1}$ & 1.04 & 1.21 & 1.54 & 1.75 & 1.64 & 1.08 & 1.27 & 1.57 & 1.76 & 1.67 \\
\hline $\mathrm{B}_{0} \mathrm{Zn}_{1} \mathrm{Fe}_{0} \mathrm{Cu}_{0}$ & 1.01 & 1.24 & 1.51 & 1.71 & 1.65 & 1.06 & 1.25 & 1.58 & 1.75 & 1.65 \\
\hline $\mathrm{B}_{0} \mathrm{Zn}_{1} \mathrm{Fe}_{0} \mathrm{Cu}_{1}$ & 1.07 & 1.25 & 1.58 & 1.77 & 1.64 & 1.11 & 1.30 & 1.59 & 1.80 & 1.69 \\
\hline $\mathrm{B}_{0} \mathrm{Zn}_{1} \mathrm{Fe}_{1} \mathrm{Cu}_{0}$ & 1.05 & 1.27 & 1.55 & 1.78 & 1.65 & 1.09 & 1.31 & 1.61 & 1.83 & 1.71 \\
\hline $\mathrm{B}_{0} \mathrm{Zn}_{1} \mathrm{Fe}_{1} \mathrm{Cu}_{1}$ & 1.14 & 1.33 & 1.62 & 1.82 & 1.74 & 1.17 & 1.38 & 1.68 & 1.88 & 1.78 \\
\hline $\mathrm{B}_{1} \mathrm{Zn}_{0} \mathrm{Fe}_{0} \mathrm{Cu}_{0}$ & 1.13 & 1.34 & 1.65 & 1.84 & 1.73 & 1.19 & 1.39 & 1.69 & 1.90 & 1.79 \\
\hline $\mathrm{B}_{1} \mathrm{Zn}_{0} \mathrm{Fe}_{0} \mathrm{Cu}_{1}$ & 1.17 & 1.36 & 1.68 & 1.86 & 1.76 & 1.20 & 1.40 & 1.70 & 1.90 & 1.80 \\
\hline $\mathrm{B}_{1} \mathrm{Zn}_{0} \mathrm{Fe}_{1} \mathrm{Cu}_{0}$ & 1.21 & 1.39 & 1.71 & 1.87 & 1.79 & 1.23 & 1.42 & 1.72 & 1.91 & 1.83 \\
\hline $\mathrm{B}_{1} \mathrm{Zn}_{0} \mathrm{Fe}_{1} \mathrm{Cu}_{1}$ & 1.26 & 1.47 & 1.77 & 1.90 & 1.78 & 1.24 & 1.43 & 1.73 & 1.95 & 1.84 \\
\hline $\mathrm{B}_{1} \mathrm{Zn}_{1} \mathrm{Fe}_{0} \mathrm{Cu}_{0}$ & 1.19 & 1.38 & 1.68 & 1.97 & 1.88 & 1.32 & 1.53 & 1.83 & 2.03 & 1.91 \\
\hline $\mathrm{B}_{1} \mathrm{Zn}_{1} \mathrm{Fe}_{0} \mathrm{Cu}_{1}$ & 1.30 & 1.50 & 1.80 & 1.99 & 1.89 & 1.35 & 1.54 & 1.84 & 2.06 & 1.94 \\
\hline $\mathrm{B}_{1} \mathrm{Zn}_{1} \mathrm{Fe}_{1} \mathrm{Cu}_{0}$ & 1.33 & 1.52 & 1.82 & 2.05 & 1.93 & 1.37 & 1.59 & 1.88 & 2.07 & 1.99 \\
\hline $\mathrm{B}_{1} \mathrm{Zn}_{1} \mathrm{Fe}_{1} \mathrm{Cu}_{1}$ & 1.37 & 1.56 & 1.85 & 2.06 & 1.96 & 1.42 & 1.64 & 1.92 & 2.13 & 2.02 \\
\hline S.Em. \pm & 2.05 & 1.74 & 1.42 & 1.27 & 1.34 & 2.18 & 1.85 & 1.53 & 1.37 & 1.44 \\
\hline C.D. at $5 \%$ & 0.29 & 0.26 & 0.23 & 0.21 & 0.22 & 3.20 & 0.27 & 0.24 & 0.21 & 0.23 \\
\hline
\end{tabular}


Table.5 Effect of pre-harvest foliar spray of micronutrients on vitamin A content at ambient storage conditions in mango fruit cv. Langra

\begin{tabular}{|c|c|c|c|c|c|c|c|c|c|c|}
\hline \multirow{3}{*}{ Treatment } & \multicolumn{10}{|c|}{ Vitamin A (mg/ $100 \mathrm{~g}$ of pulp) } \\
\hline & \multicolumn{5}{|c|}{ 2004-05 } & \multicolumn{5}{|c|}{ 2005-06 } \\
\hline & 0 day & 2 days & 4 days & 6 days & 8 days & 0 day & 2 days & 4 days & 6 days & 8 days \\
\hline $\mathrm{B}_{0} \mathrm{Zn}_{0} \mathrm{Fe}_{0} \mathrm{Cu}_{0}$ & 1.58 & 1.90 & 2.40 & 2.72 & 2.57 & 1.60 & 1.97 & 2.48 & 2.80 & 2.62 \\
\hline $\mathrm{B}_{0} \mathrm{Zn}_{0} \mathrm{Fe}_{0} \mathrm{Cu}_{1}$ & 1.65 & 1.98 & 2.50 & 2.82 & 2.67 & 1.70 & 2.05 & 2.53 & 2.88 & 2.72 \\
\hline $\mathrm{B}_{0} \mathrm{Zn}_{0} \mathrm{Fe}_{1} \mathrm{Cu}_{0}$ & 1.67 & 2.00 & 2.48 & 2.83 & 2.65 & 1.72 & 2.07 & 2.58 & 2.88 & 2.73 \\
\hline $\mathrm{B}_{0} \mathrm{Zn}_{0} \mathrm{Fe}_{1} \mathrm{Cu}_{1}$ & 1.73 & 2.02 & 2.57 & 2.92 & 2.73 & 1.80 & 2.12 & 2.62 & 2.93 & 2.78 \\
\hline $\mathrm{B}_{0} \mathrm{Zn}_{1} \mathrm{Fe}_{0} \mathrm{Cu}_{0}$ & 1.68 & 2.07 & 2.52 & 2.85 & 2.75 & 1.77 & 2.08 & 2.63 & 2.92 & 2.75 \\
\hline $\mathrm{B}_{0} \mathrm{Zn}_{1} \mathrm{Fe}_{0} \mathrm{Cu}_{1}$ & 1.78 & 2.08 & 2.63 & 2.95 & 2.73 & 1.85 & 2.17 & 2.65 & 3.00 & 2.82 \\
\hline $\mathrm{B}_{0} \mathrm{Zn}_{1} \mathrm{Fe}_{1} \mathrm{Cu}_{0}$ & 1.75 & 2.12 & 2.58 & 2.97 & 2.75 & 1.82 & 2.18 & 2.68 & 3.05 & 2.85 \\
\hline $\mathrm{B}_{0} \mathrm{Zn}_{1} \mathrm{Fe}_{1} \mathrm{Cu}_{1}$ & 1.90 & 2.22 & 2.70 & 3.03 & 2.90 & 1.95 & 2.30 & 2.80 & 3.13 & 2.97 \\
\hline $\mathrm{B}_{1} \mathrm{Zn}_{0} \mathrm{Fe}_{0} \mathrm{Cu}_{0}$ & 1.88 & 2.23 & 2.75 & 3.07 & 2.88 & 1.98 & 2.32 & 2.82 & 3.17 & 2.98 \\
\hline $\mathrm{B}_{1} \mathrm{Zn}_{0} \mathrm{Fe}_{0} \mathrm{Cu}_{1}$ & 1.95 & 2.27 & 2.80 & 3.10 & 2.93 & 2.00 & 2.33 & 2.83 & 3.17 & 3.00 \\
\hline $\mathrm{B}_{1} \mathrm{Zn}_{0} \mathrm{Fe}_{1} \mathrm{Cu}_{0}$ & 2.02 & 2.32 & 2.85 & 3.12 & 2.98 & 2.05 & 2.37 & 2.87 & 3.18 & 3.05 \\
\hline $\mathrm{B}_{1} \mathrm{Zn}_{0} \mathrm{Fe}_{1} \mathrm{Cu}_{1}$ & 2.10 & 2.45 & 2.95 & 3.17 & 2.97 & 2.07 & 2.38 & 2.88 & 3.25 & 3.07 \\
\hline $\mathrm{B}_{1} \mathrm{Zn}_{1} \mathrm{Fe}_{0} \mathrm{Cu}_{0}$ & 1.98 & 2.30 & 2.80 & 3.28 & 3.13 & 2.20 & 2.55 & 3.05 & 3.38 & 3.18 \\
\hline $\mathrm{B}_{1} \mathrm{Zn}_{1} \mathrm{Fe}_{0} \mathrm{Cu}_{1}$ & 2.17 & 2.50 & 3.00 & 3.32 & 3.15 & 2.25 & 2.57 & 3.07 & 3.43 & 3.23 \\
\hline $\mathrm{B}_{1} \mathrm{Zn}_{1} \mathrm{Fe}_{1} \mathrm{Cu}_{0}$ & 2.22 & 2.53 & 3.03 & 3.42 & 3.22 & 2.28 & 2.65 & 3.13 & 3.45 & 3.32 \\
\hline $\mathrm{B}_{1} \mathrm{Zn}_{1} \mathrm{Fe}_{1} \mathrm{Cu}_{1}$ & 2.28 & 2.60 & 3.08 & 3.43 & 3.27 & 2.37 & 2.73 & 3.20 & 3.55 & 3.37 \\
\hline S.Em. \pm & 2.16 & 1.78 & 1.42 & 3.69 & 4.43 & 2.20 & 1.89 & 1.66 & 1.38 & 1.63 \\
\hline C.D. at $5 \%$ & 0.34 & 0.23 & 0.21 & 0.57 & 0.61 & 0.35 & 0.24 & 0.24 & 0.21 & 0.23 \\
\hline
\end{tabular}


Table.6 Effect of pre-harvest foliar spray of micronutrients on TSS : acid ratio at ambient storage conditions in mango fruit cv. Langra

\begin{tabular}{|c|c|c|c|c|c|c|c|c|c|c|}
\hline \multirow{3}{*}{ Treatment } & \multicolumn{10}{|c|}{ TSS : Acid ratio } \\
\hline & \multicolumn{5}{|c|}{ 2004-05 } & \multicolumn{5}{|c|}{ 2005-06 } \\
\hline & 0 day & 2 days & 4 days & 6 days & 8 days & 0 day & 2 days & 4 days & 6 days & 8 days \\
\hline $\mathrm{B}_{0} \mathrm{Zn}_{0} \mathrm{Fe}_{0} \mathrm{Cu}_{0}$ & $1: 0.0130$ & $1: 0.0112$ & $1: 0.0091$ & $1: 0.0096$ & $1: 0.0237$ & $1: 0.0130$ & $1: 0.0112$ & $1: 0.0091$ & $1: 0.0097$ & $1: 0.0236$ \\
\hline $\mathrm{B}_{0} \mathrm{Zn}_{0} \mathrm{Fe}_{0} \mathrm{Cu}_{1}$ & 1:0.0130 & $1: 0.0112$ & $1: 0.0083$ & $1: 0.0093$ & $1: 0.0236$ & $1: 0.0127$ & 1:0.0109 & $1: 0.0089$ & $1: 0.0094$ & 1:0.0232 \\
\hline $\mathrm{B}_{0} \mathrm{Zn}_{0} \mathrm{Fe}_{1} \mathrm{Cu}_{0}$ & 1:0.0127 & 1:0.0108 & $1: 0.0091$ & $1: 0.0092$ & $1: 0.0236$ & $1: 0.0126$ & 1:0.0108 & $1: 0.0087$ & $1: 0.0090$ & $1: 0.0231$ \\
\hline $\mathrm{B}_{0} \mathrm{Zn}_{0} \mathrm{Fe}_{1} \mathrm{Cu}_{1}$ & 1:0.0120 & 1:0.0096 & $1: 0.0087$ & $1: 0.0090$ & $1: 0.0235$ & $1: 0.0120$ & $1: 0.0103$ & $1: 0.0084$ & $1: 0.0086$ & 1:0.0229 \\
\hline $\mathrm{B}_{0} \mathrm{Zn}_{1} \mathrm{Fe}_{0} \mathrm{Cu}_{0}$ & $1: 0.0125$ & 1:0.0103 & 1:0.0086 & $1: 0.0086$ & $1: 0.0226$ & 1:0.0120 & 1:0.0102 & 1:0.0082 & 1:0.0086 & $1: 0.0224$ \\
\hline $\mathrm{B}_{0} \mathrm{Zn}_{1} \mathrm{Fe}_{0} \mathrm{Cu}_{1}$ & $1: 0.0125$ & 1:0.0106 & $1: 0.0085$ & $1: 0.0083$ & $1: 0.0221$ & $1: 0.0118$ & $1: 0.0101$ & $1: 0.0081$ & $1: 0.0086$ & 1:0.0221 \\
\hline $\mathrm{B}_{0} \mathrm{Zn}_{1} \mathrm{Fe}_{1} \mathrm{Cu}_{0}$ & $1: 0.0115$ & 1:0.0091 & $1: 0.0078$ & $1: 0.0081$ & $1: 0.0221$ & $1: 0.0115$ & 1:0.0099 & 1:0.0080 & 1:0.0084 & $1: 0.0217$ \\
\hline $\mathrm{B}_{0} \mathrm{Zn}_{1} \mathrm{Fe}_{1} \mathrm{Cu}_{1}$ & 1:0.0113 & 1:0.0098 & $1: 0.0074$ & 1:0.0079 & $1: 0.0215$ & $1: 0.0114$ & 1:0.0099 & $1: 0.0077$ & $1: 0.0081$ & 1:0.0218 \\
\hline $\mathrm{B}_{1} \mathrm{Zn}_{0} \mathrm{Fe}_{0} \mathrm{Cu}_{0}$ & $1: 0.0125$ & 1:0.0107 & 1:0.0086 & $1: 0.0075$ & $1: 0.0214$ & 1:0.0112 & 1:0.0091 & 1:0.0075 & 1:0.0077 & 1:0.0217 \\
\hline $\mathrm{B}_{1} \mathrm{Zn}_{0} \mathrm{Fe}_{0} \mathrm{Cu}_{1}$ & $1: 0.0114$ & 1:0.0096 & 1:0.0076 & $1: 0.0073$ & $1: 0.0221$ & 1:0.0114 & 1:0.0094 & 1:0.0074 & 1:0.0076 & 1:0.0213 \\
\hline $\mathrm{B}_{1} \mathrm{Zn}_{0} \mathrm{Fe}_{1} \mathrm{Cu}_{0}$ & 1:0.0117 & 1:0.0090 & $1: 0.0077$ & $1: 0.0070$ & $1: 0.0211$ & 1:0.0108 & 1:0.0091 & $1: 0.0073$ & $1: 0.0075$ & 1:0.0212 \\
\hline $\mathrm{B}_{1} \mathrm{Zn}_{0} \mathrm{Fe}_{1} \mathrm{Cu}_{1}$ & $1: 0.0114$ & 1:0.0099 & 1:0.0073 & 1:0.0075 & $1: 0.0218$ & 1:0.0107 & 1:0.0093 & 1:0.0072 & 1:0.0075 & 1:0.0207 \\
\hline $\mathrm{B}_{1} \mathrm{Zn}_{1} \mathrm{Fe}_{0} \mathrm{Cu}_{0}$ & 1:0.0110 & 1:0.0093 & $1: 0.0071$ & $1: 0.0073$ & $1: 0.0211$ & 1:0.0113 & 1:0.0092 & $1: 0.0071$ & $1: 0.0073$ & 1:0.0201 \\
\hline $\mathrm{B}_{1} \mathrm{Zn}_{1} \mathrm{Fe}_{0} \mathrm{Cu}_{1}$ & 1:0.0100 & 1:0.0096 & $1: 0.0078$ & $1: 0.0070$ & $1: 0.0192$ & 1:0.0106 & 1:0.0090 & 1:0.0071 & $1: 0.0073$ & $1: 0.0200$ \\
\hline $\mathrm{B}_{1} \mathrm{Zn}_{1} \mathrm{Fe}_{1} \mathrm{Cu}_{0}$ & 1:0.0107 & 1:0.0085 & 1:0.0067 & $1: 0.0066$ & $1: 0.0191$ & 1:0.0095 & 1:0.0080 & 1:0.0064 & $1: 0.0062$ & $1: 0.0181$ \\
\hline $\mathrm{B}_{1} \mathrm{Zn}_{1} \mathrm{Fe}_{1} \mathrm{Cu}_{1}$ & 1:0.0094 & 1:0.0080 & 1:0.0064 & $1: 0.0062$ & $1: 0.0181$ & 1:0.0094 & 1:0.0079 & 1:0.0063 & 1:0.0061 & 1:0.0180 \\
\hline
\end{tabular}


Table.7 Effect of pre-harvest foliar spray of micronutrients on sugar : acid ratio at ambient storage conditions in mango fruit cv. Langra

\begin{tabular}{|c|c|c|c|c|c|c|c|c|c|c|}
\hline \multirow{3}{*}{ Treatment } & \multicolumn{10}{|c|}{ Sugar : Acid ratio } \\
\hline & \multicolumn{5}{|c|}{ 2004-05 } & \multicolumn{5}{|c|}{ 2005-06 } \\
\hline & 0 day & 2 days & 4 days & 6 days & 8 days & 0 day & 2 days & 4 days & 6 days & 8 days \\
\hline $\mathrm{B}_{0} \mathrm{Zn}_{0} \mathrm{Fe}_{0} \mathrm{Cu}_{0}$ & $1: 0.0246$ & $1: 0.0222$ & 1:0.0196 & 1:0.0195 & $1: 0.0428$ & $1: 0.0232$ & 1:0.0209 & $1: 0.0184$ & 1:0.0181 & 1:0.0403 \\
\hline $\mathrm{B}_{0} \mathrm{Zn}_{0} \mathrm{Fe}_{0} \mathrm{Cu}_{1}$ & $1: 0.0232$ & 1:0.0209 & $1: 0.0185$ & 1:0.0180 & 1:0.0403 & $1: 0.0220$ & 1:0.0198 & $1: 0.0174$ & $1: 0.0170$ & $1: 0.0388$ \\
\hline $\mathrm{B}_{0} \mathrm{Zn}_{0} \mathrm{Fe}_{1} \mathrm{Cu}_{0}$ & $1: 0.0226$ & 1:0.0203 & 1:0.0178 & 1:0.0174 & $1: 0.0400$ & $1: 0.0211$ & 1:0.0190 & 1:0.0168 & 1:0.0168 & $1: 0.0369$ \\
\hline $\mathrm{B}_{0} \mathrm{Zn}_{0} \mathrm{Fe}_{1} \mathrm{Cu}_{1}$ & $1: 0.0218$ & 1:0.0196 & $1: 0.0172$ & 1:0.0169 & 1:0.0388 & 1:0.0203 & $1: 0.0188$ & $1: 0.0160$ & 1:0.0159 & $1: 0.0369$ \\
\hline $\mathrm{B}_{0} \mathrm{Zn}_{1} \mathrm{Fe}_{0} \mathrm{Cu}_{0}$ & 1:0.0206 & $1: 0.0184$ & $1: 0.0162$ & 1:0.0161 & $1: 0.0370$ & 1:0.0202 & $1: 0.0182$ & 1:0.0159 & 1:0.0157 & $1: 0.0367$ \\
\hline $\mathrm{B}_{0} \mathrm{Zn}_{1} \mathrm{Fe}_{0} \mathrm{Cu}_{1}$ & 1:0.0203 & 1:0.0181 & $1: 0.0159$ & 1:0.0158 & 1:0.0369 & 1:0.0201 & 1:0.0179 & $1: 0.0156$ & $1: 0.0155$ & 1:0.0366 \\
\hline $\mathrm{B}_{0} \mathrm{Zn}_{1} \mathrm{Fe}_{1} \mathrm{Cu}_{0}$ & 1:0.0202 & $1: 0.0182$ & 1:0.0158 & 1:0.0158 & $1: 0.0368$ & 1:0.0201 & 1:0.0179 & 1:0.0156 & $1: 0.0153$ & $1: 0.0358$ \\
\hline $\mathrm{B}_{0} \mathrm{Zn}_{1} \mathrm{Fe}_{1} \mathrm{Cu}_{1}$ & $1: 0.0201$ & $1: 0.0180$ & $1: 0.0158$ & 1:0.0154 & 1:0.0366 & $1: 0.0185$ & $1: 0.0165$ & 1:0.0145 & $1: 0.0145$ & $1: 0.0336$ \\
\hline $\mathrm{B}_{1} \mathrm{Zn}_{0} \mathrm{Fe}_{0} \mathrm{Cu}_{0}$ & $1: 0.0200$ & $1: 0.0178$ & 1:0.0156 & 1:0.0152 & 1:0.0366 & $1: 0.0183$ & 1:0.0159 & 1:0.0136 & 1:0.0139 & 1:0.0331 \\
\hline $\mathrm{B}_{1} \mathrm{Zn}_{0} \mathrm{Fe}_{0} \mathrm{Cu}_{1}$ & 1:0.0186 & 1:0.0165 & $1: 0.0143$ & 1:0.0139 & $1: 0.0345$ & 1:0.0182 & 1:0.0158 & $1: 0.0121$ & 1:0.0132 & 1:0.0333 \\
\hline $\mathrm{B}_{1} \mathrm{Zn}_{0} \mathrm{Fe}_{1} \mathrm{Cu}_{0}$ & $1: 0.0183$ & $1: 0.0162$ & $1: 0.0138$ & 1:0.0136 & 1:0.0337 & $1: 0.0179$ & $1: 0.0157$ & 1:0.0135 & 1:0.0131 & $1: 0.0330$ \\
\hline $\mathrm{B}_{1} \mathrm{Zn}_{0} \mathrm{Fe}_{1} \mathrm{Cu}_{1}$ & $1: 0.0182$ & $1: 0.0162$ & 1:0.0135 & 1:0.0136 & $1: 0.0336$ & $1: 0.0178$ & 1:0.0149 & 1:0.0135 & 1:0.0130 & $1: 0.0324$ \\
\hline $\mathrm{B}_{1} \mathrm{Zn}_{1} \mathrm{Fe}_{0} \mathrm{Cu}_{0}$ & 1:0.0178 & 1:0.0160 & 1:0.0141 & 1:0.0133 & 1:0.0336 & 1:0.0164 & 1:0.0156 & 1:0.0125 & 1:0.0118 & 1:0.0301 \\
\hline $\mathrm{B}_{1} \mathrm{Zn}_{1} \mathrm{Fe}_{0} \mathrm{Cu}_{1}$ & 1:0.0170 & 1:0.0157 & $1: 0.0140$ & 1:0.0132 & 1:0.0335 & 1:0.0160 & 1:0.0145 & $1: 0.0122$ & 1:0.0118 & 1:0.0297 \\
\hline $\mathrm{B}_{1} \mathrm{Zn}_{1} \mathrm{Fe}_{1} \mathrm{Cu}_{0}$ & 1:0.0169 & 1:0.0150 & 1:0.0130 & 1:0.0120 & 1:0.0314 & 1:0.0157 & 1:0.0142 & $1: 0.0121$ & 1:0.0115 & $1: 0.0290$ \\
\hline $\mathrm{B}_{1} \mathrm{Zn}_{1} \mathrm{Fe}_{1} \mathrm{Cu}_{1}$ & 1:0.0164 & 1:0.0145 & $1: 0.0125$ & 1:0.0115 & 1:0.0301 & 1:0.0149 & 1:0.0132 & $1: 0.0114$ & 1:0.0105 & 1:0.0276 \\
\hline
\end{tabular}


A positive coefficient of correlation (0.977) existed between total sugar and TSS contents. The acidity and ascorbic acid contents showed a declining trend, although the later showed some increase in the initial stages of storage (Kumar, 2009). Similarly T.S.S. increased initially up to 3 days with a subsequent decline thereafter irrespective of cultivars of Ber (Sanches et al., 2005). The harvested fruits were stored at an ambient temperature (ATS) of $25 \pm 2{ }^{\circ} \mathrm{C}$ and at low temperature storage (LTS) of $15 \pm 2^{\circ} \mathrm{C}$ with $60 \%-70 \%$ relative humidity (RH) for 60 days. Sweet oranges stored at LTS maintained better fruit quality than ATS. The foliar application of zinc and boron significantly enhanced fruit juice content, total soluble solids (TSS), ascorbic acid (AA) and nonreducing sugar (NRS) of fruit. However, fruit juice content, TSS and AA were observed significantly higher, when the fruit was treated with high zinc (1\%) and low boron $(0.02 \%)$. The percent of weight loss, disease incidence, TSS and reducing sugar (RS) increased with increasing the storage durations. A reduction was noted in fruit juice, AA and NRS with increasing the storage durations (Sajid et al., 2012).

Data showed that there was minimum $\beta$ carotene content in the fruits on the day of harvest under all the treatments and it showed an increasing trend up to $6^{\text {th }}$ day of storage period than it declined in both the years. Minimum $\beta$-carotene content was recorded in control treatment and maximum in the treatment having combination of all four micronutrients application and the difference was significant between minimum and maximum values of $\beta$-carotene of the fruits stored up to $8^{\text {th }}$ days in the first and second year (Table 4).

Vitamin A contents showed a continuous increase throughout irrespective of the treatments applied. It was concluded that, for maintaining an overall high quality of fruits at room storage conditions for 8-10 days, two pre-harvest foliar sprays of 1 per cent aqueous solution of zinc sulphate ( $\mathrm{Zn} 0.23$ per cent) at 20 days interval in May were found to be quite beneficial (Kumar, 2009). Similarly Kowsalya and Chandrasekhar (2003) suggested that in papaya fruit total carotenoid and $\beta$-carotene contents from the greenish yellow stage to fully ripened stage (after 24 hours storage at room temperature) significantly increased. The fully ripened papaya when stored further at room temperature for 2 days showed significant reduction in total carotenoid and $\beta$-carotene contents. Among the mango varieties studied, total carotenoid and $\beta$-carotene contents in ripe fruits were maximum in Malgoa and Banganpally varieties, followed by Naducholar and Neelam. Jack fruit cv. Halwa only retained minimal total carotenoid and $\beta$ carotene contents. Data on vitamin A content of the fruit stored at room temperature was calculated on the basis of recorded $\beta$-carotene content in table 5. The similar trend of vitamin A was observed as the $\beta$-carotene content in the both the years (Table 5). Kowsalya and Chandrasekhar (2003) also supported research findings on vitamin A content.

TSS : acid ratio was found minimum in the treatment combination having foliar application of all the four micronutrients followed by treatment combination of $\mathrm{B}+\mathrm{Zn}$ $+\mathrm{Cu}$ and $\mathrm{B}+\mathrm{Zn}+\mathrm{Fe}$. Ratio was maximum under control followed by treatment having only copper on the different days of the storage period in the first and second year. Data also revealed that TSS : acid ratio decrease up to $6^{\text {th }}$ day of storage under all the treatments and then it increased in both the years (Table 6). Sanches et al., (2005) reported that TSS : acid ratio increased throughout the storage period. Mean maximum values of TSS : acid ratio was 
observed irrespective of cultivars. Data in table 7, showed that at room temperature conditions, sugar : acid ratio was maximum in control fruit followed by the treatment having only copper application from the day of harvest to $8^{\text {th }}$ day of storage period while it was minimum in treatment combination having boron, zinc, iron and copper followed by the treatment having boron, zinc and iron only in the first and second year. Data also revealed that sugar : acid ratio declined up to $6^{\text {th }}$ day of storage period than it increased in both the years. Similarly, Hoda et al., (1975) and Kumar et al., (1995) found an increase in total sugars, ascorbic acid and sugar : acid ratio in litchi fruits with foliar application of zinc sulphate (Table 7).

\section{References}

De Candolle (1904). Origin of cultivated plants. Kegan Paul, London.

Hoda, M.N.; Syamal, N.B. and Chhonker, V.S. (1975). Growth substances and zinc on the development of quality of litchi fruits. Sci. \& Cult., 35(9): 440448.

Indian Horticulture Data Base, 2010-11.

Indian Horticulture Data Base, 2011-12.

Kowsalya, S. and Chandrasekhar, U. (2003). Total carotenoid and beta-carotene contents of selected stored plant foods. Indian Journal of Nutrition and Dietetics, 40(4): 122-128.
Kumar, O.V. and Kumar, G. (2009). Effect of pre-harvest foliar sprays of zinc on post-harvest changes in the quality of mango cv dashehari. Acta horticulturae. 231.

Sajid, Muhammad,; Rab, Abdur,; Jan, Ibadullah,; Haq., Ihsanul; Shah,Syed Tanveer,; Iqbal, Amjad,; Zamin, Muhammad and Muhammad, Shakur. (2012). Pre-harvest Treatment of Zn and B Affects the Fruit Quality and Storability of Sweet Orange. Journal of Agricultural Science and Technology, ISSN 1939-1250. B 2: 1224-1233

Sanches, J.; Leal, P.A.M.; Saravali, J.H. and Antoniali, S. (2005). Principal components analysis for quality evaluation of cooled banana 'Nanicao' in different packing. Revista Brasileira-de-Fruticultura, $\quad$ 25(2): 220-223.

Singh, Rajbir.; Sharma, R.R. and Tyagi, S.K. (2007). Pre-harvest foliar application of calcium and boron influences physiological disorders, fruit yield and quality of strawberry (Fragaria $\times$ ananassa Duch.). Scientia Horticulture., 112(2):215-220.

Srivastava, R.P. (1993). Fruits and vegetable Preservation International Book Distributing Co. Publishing Division, Luck now. 444 p.

\section{How to cite this article:}

Rashmi Pawar C.P. Singh, P.P. Singh and Meena, R. 2017. Effect of Pre-Harvest Foliar Spray of Micronutrients on Chemical Properties of Mango Cv. Langra at Ambient Storage Conditions. Int.J.Curr.Microbiol.App.Sci. 6(4): 2557-2567. doi: https://doi.org/10.20546/ijcmas.2017.604.298 